Cahiers $d u$ MONDE RUSSE

\section{Cahiers du monde russe}

Russie - Empire russe - Union soviétique et États indépendants

55/3-4 | 2014

Varia

\title{
Charles Stepanoff, Carole Ferret, Gaëlle Lacaze, Julien Thorez, éds., Nomadismes d'Asie centrale et septentrionale
}

Joëlle Robert-Lamblin

\section{(2) OpenEdition}

\section{Édition électronique}

URL : http://journals.openedition.org/monderusse/8126

DOI : $10.4000 /$ monderusse. 8126

ISSN : $1777-5388$

Éditeur

Éditions de l'EHESS

\section{Édition imprimée}

Date de publication : 1 juillet 2014

Pagination : 491-496

ISBN : 978-2-7132-2441-6

ISSN : $1252-6576$

\section{Référence électronique}

Joëlle Robert-Lamblin, « Charles Stepanoff, Carole Ferret, Gaëlle Lacaze, Julien Thorez, éds., Nomadismes d'Asie centrale et septentrionale », Cahiers du monde russe [En ligne], 55/3-4 | 2014, mis en ligne le 14 avril 2015, Consulté le 25 septembre 2020. URL : http://journals.openedition.org/ monderusse/8126; DOI : https://doi.org/10.4000/monderusse.8126

Ce document a été généré automatiquement le 25 septembre 2020.

(c) École des hautes études en sciences sociales 


\title{
Charles Stepanoff, Carole Ferret, Gaëlle Lacaze, Julien Thorez, éds., Nomadismes d'Asie centrale et septentrionale
}

\author{
Joëlle Robert-Lamblin
}

\section{RÉFÉRENCE}

Charles STEPANOFF, Carole FERRET, Gaëlle LACAZE, Julien THOREZ, éds., Nomadismes d'Asie centrale et septentrionale, Paris : Armand Colin, 2013, 288 p.

1 Le thème du nomadisme, traité à dessein au pluriel plutôt qu'au singulier, tant il est vrai que ce terme recouvre des situations très diverses, suscite de nos jours intérêt et curiosité. Très pédagogique, comprenant des encadrés pour détailler un point particulier, émaillé de nombreuses références, de documents d'archives et très bien illustré par des cartes, photos et schémas, cet ouvrage est le fruit d'une collaboration internationale réunissant trente-neuf contributeurs: anthropologues, géographes, archéologues et historiens, de degrés universitaires divers. Outre la richesse des informations scientifiques qu'il contient et détaille, ce livre reste accessible à un large public en étant tout autant agréable à lire exhaustivement qu'à consulter ponctuellement.

2 Le sujet recouvre une région géographique particulièrement vaste : la partie asiatique de l'ancienne URSS, la république de Mongolie et le nord de la Chine; il relevait donc du défi d'éviter la présentation d'une sorte de catalogue des différentes formes de mobilité existant dans le temps ou dans l'espace.

Dans l'introduction, Charles Stépanoff souligne la diversité des nomadismes contemporains. Citant Brian Spooner, il précise qu'il n'y a rien de commun à tous les nomades ni rien d'exclusif chez les nomades. Il donne une définition du nomadisme : 
un « mode de vie mobile, impliquant des familles [...], partagé avec des animaux dont le comportement et les besoins jouent un rôle central dans les déplacements, [...] marqué par un lien paradoxal à l'espace fait d'une conjonction de maîtrise et de retrait» (p. 8). À travers des synthèses et des études de cas réalisées sur le terrain par les chercheurs, le lecteur découvrira « les savoirs, les modes de perception du monde, les adaptations et le dynamisme des populations nomades et anciennement nomades d'Asie centrale et septentrionale». Pour leur part, Gaëlle Lacaze et Julien Thorez replacent les populations concernées dans leur contexte géographique et historique et indiquent leur statut actuel. Ils soulignent également que ces peuples ont en commun d'avoir été colonisés par les Empires chinois ou russe, puis d'avoir subi la "soviétisation", elle-même suivie par la période des privatisations qui mit fin à la collectivisation.

4 L'ouvrage se compose de cinq parties, presque équivalentes en taille, respectivement intitulées: «milieux et mobilités», "habiter le territoire», "espace et temps du corps ", "hommes et bêtes en mouvement" et, enfin, "nomades et sédentaires". Chaque partie est introduite par un texte synthétique qui présente l'essentiel de son contenu et de courts résumés précèdent les différents sous-chapitres.

Dans le nomadisme, hommes, bétails et milieux interagissent constamment, c'est pourquoi la première partie du livre est tout naturellement consacrée à une description à grands traits des différents écosystèmes: toundra, taïga, steppes, régions montagneuses, et des «systèmes nomades » qui sont liés à ces milieux : pastoralisme dominant dans steppe et toundra, élevage associé à la chasse et à la pêche dans la taïga. Plusieurs analyses détaillées font ressortir les particularités du pastoralisme kazakh (C. Ferret) et mongol (C. Marchina), de même que celui de la toundra et de la taïga sibériennes où nomadisent les Nénetses (A. Golovnev) et les Evenks (A. Lavriller). La division de l'année, prenant en compte les savoirs sur l'écosystème et le climat local, a été à la source de création de multiples calendriers ponctuant les activités annuelles des nomades dans les différentes régions. Des exemples d'itinéraires de déplacements dans l'espace, illustrés par des cartes ou des schémas très bien conçus (notamment ceux de C. Fossier et J.-C. Raynal pour les Evènes du Kamtchatka), facilitent grandement la compréhension des mouvements migratoires complexes combinant changements de latitude et d'altitude au cours du cycle annuel de migration. Certains sont encore en usage, d'autres se réfèrent à des périodes précédant la collectivisation ou lors de la transition postsoviétique. Cette partie s'achève sur la présentation des «techniques du mouvement", à savoir les moyens de transport, les modes d'orientation et l'organisation sociale de la mobilité. Il est à noter que les caravanes, composées d'humains, d'animaux de transport, d'équipements et de troupeaux, reproduisent la hiérarchie sociale de l'organisation de l'espace du campement chez les Evenks (A. Lavriller).

6 La deuxième partie du livre, "habiter le territoire ", est consacrée à l'usage quotidien de l'espace par les nomades, le temps passé dans un campement et les lieux environnants, ainsi qu'à l'organisation des espaces aménagés et domestiques. Si la propriété des lieux est le plus souvent reconnue aux esprits (qu'il faut respecter, vénérer et honorer par rituels et offrandes), les nomades disposent pour leur part de droits d'usage et de passage, ainsi que de droits de pacage et d'accès aux territoires de chasse, ces droits collectifs étant héréditaires par patrilinéarité. Concernant les habitations proprement dites, sont présentées successivement les spécificités de la construction et de l'aménagement intérieur, de la yaranga tchouktche (V. Vaté), de la 
yourte turco-mogole (I. Oktiabrskaïa, C. Lacaze et Ch. Stépanoff), de la maison de bois de Sibérie du Sud (Ch. Stépanoff) habitée par des nomades

semi-sédentarisés, et d'autres modèles encore. La tente nomade - figurant le ciel et la terre - symbolise l'univers, tandis qu'à l'intérieur, l'espace s'organise selon les rapports sociaux : espace public (lieu de passage ou de travail) et espace privé (où l'on dort) se distinguent, de même que parfois espaces masculins et féminins sont strictement divisés, comme le sont les activités domestiques et religieuses, notamment chez les Nénetses (A. Golovnev). Sur le campement nomade, les tentes sont disposées en fonction de la hiérarchie existante entre aînés et cadets, riches et pauvres, etc. Les espaces sacrés, qu'ils soient au sein de l'habitation, dans le campement, ou à l'extérieur de celui-ci, jouent un rôle primordial. Des conduites et des rituels différents sont prescrits aux hommes et aux femmes ; à cet égard, l'exemple du découpage de l'espace dans l'aul kazakh et ses alentours, décrit et analysé par S. Aouelbekov et C. Ferret, est particulièrement intéressant. Cette partie s'achève en mettant en relief l'importance de l'hospitalité, qualifiée de fondement des relations sociales chez les nomades (I. Oktiabrskaïa, C. Lacaze et A. Golovnev), et sur l'évocation de grands rassemblements saisonniers donnant lieu à des jeux rituels, des compétitions sportives et de nombreux échanges, chez les Tchouktches (V. Vaté) et plus développés encore chez les Mongols lors de la fête de l'été (G. Lacaze).

7 Vient ensuite un ensemble de textes consacrés à "l'espace et le temps du corps", partie un peu touffue où sont abordés le positionnement du corps dans le temps et l'espace avec les oppositions devant/derrière, haut/bas, gauche/droite, que l'on trouve dans l'organisation domestique nomade. Puis est évoqué l'apprentissage des savoirs et des conduites au cours de la vie. Par l'éducation qui lui est prodiguée, l'enfant nomade devra "acquérir le savoir-être et le savoir-faire destinés à devenir des habitus ", selon Marcel Mauss dans son manuel «Les Techniques du corps ». L'enfant se construit en observant et en participant progressivement aux tâches collectives. Il devient très vite indépendant et responsable ; il est incité à savoir se maîtriser et contenir ses émotions. Parmi les exemples décrits, on trouvera les étapes de la vie chez les Nénetses (A. Golovnev), le trajet mongol pour découvrir le monde (G. Lacaze et A. Michelet), la transmission sexuée chez les Tchouktches (V. Vaté). Dans la conception du corps chez les Mongols (G.Lacaze), on apprend que celui-ci est constitué de deux éléments fondamentaux : les os hérités du père et la chair transmise par la mère; ceux-ci sont complémentaires et fondent les conceptions de la personne. C'est au cours de la petite enfance que le corps doit se "fermer " pour que l'âme puisse s'y fixer définitivement. La première coupe de cheveux chez divers nomades mongols revêt une grande importance car elle va de pair avec l'acquisition d'une identité sexuée et se trouve en outre mise en parallèle avec la taille de la crinière des poulains. Les «techniques du corps " sont encore associées à l'esthétique dans ce qui a trait à la gestuelle, aux mouvements, et aux vêtements - prolongements du corps - dont les modèles et ornements constituent également des marqueurs sociaux. Dans la sous-partie dédiée à la perception du monde, est passé en revue ce qui a trait aux sens des individus: odorat, ouïe, vue, goût, si essentiels pour interpréter les divers messages de la nature. En effet chaque changement de saison est marqué par des modifications sonores, visuelles et olfactives du paysage. Viennent illustrer cette rubrique : cuisine et goûts alimentaires des pasteurs (G. Lacaze et C. Ferret), les différentes notions de son et de bruit chez les Mongols (L. Legrain), ainsi que le «calendrier sonore» des nomades touvas, fondé sur les divers chants d'oiseaux et cris de mammifères qui sont les signes 
avant-coureurs des changements de saison avec une indication pour les hommes d'entreprendre certaines activités. Pour finir, dans "chamanisme et nomadisme", Ch. Stépanoff montre la façon dont les chamanes conduisent leurs rituels en voyageant virtuellement à travers divers mondes, sans jamais perdre contact avec le public.

La quatrième partie, " hommes et bêtes en mouvement ", met en évidence à quel point nourrissage et gardiennage du bétail peuvent varier selon les espèces, le temps et les lieux. Les rythmes de nomadisation sont ainsi très divers, selon qu'il s'agit par exemple, d'élevage de rennes ou de chevaux dont les modes de gardiennage diffèrent grandement. Il en est de même, en Asie centrale, pour ce qui a trait au gros bétail (bovins, chevaux, chameaux) et au petit bétail (moutons et chèvres). En démonstration de cela, on appréciera les intéressantes figures des pages 170-171 présentant la répartition des divers types d'élevage dans la partie asiatique de la Russie, en Mongolie et au Kazakhstan, ainsi que deux modèles différents de calendriers d'activités d'élevage et de déplacements saisonniers relevés au Kazakhstan et au Kamtchatka. Les troupeaux d'animaux cités se répartissent entre élevages mono spécifiques, comme celui du renne en Sibérie, et élevages pluri spécifiques comme ceux qui sont pratiqués en Asie centrale, Mongolie et Sibérie méridionale, comportant plusieurs espèces de gros et de petit bétail, avec l'exemple de l'élevage mongol décrit par C. Marchina. La multiplicité d'élevages, quoique contraignante, permet de se prémunir contre les aléas susceptibles de toucher certaines espèces : épizootie ou catastrophes climatiques de type zud. La diversité des élevages mentionnés comprend avant tout le cheval, dont C. Ferret précise qu'avec le renne c'est « l'espèce la plus emblématique du nomadisme pastoral en Asie centrale et septentrionale ». Selon cet auteur, "une civilisation du cheval qui imprègne tous les pans de la culture s'est développée chez les peuples cavaliers altaïques». Pour les rennes, deux types d'élevage existent en Sibérie : les grands troupeaux de la toundra, très mobiles, et comportant plusieurs centaines ou milliers d'animaux destinés à la production de viande, et les petits troupeaux de la taïga essentiellement dédiés au transport et à la production de lait (Ch. Stépanoff). Qu'il s'agisse des rennes de Sibérie ou des ovins et des bovins du Kirghizstan, une constante se retrouve pour les années 1990: la désorganisation des structures collectives et l'effondrement des débouchés ont entrâné une diminution dramatique des cheptels. Les " animaux au singulier» désignent ceux avec lesquels les hommes nouent des relations particulières : tels les rennes de selle ou de trait des Evènes, les chevaux partenaires de travail des pasteurs mongols, ou encore les chiens des éleveurs de rennes tchouktches, gardiens du campement et du troupeau. Enfin, certains animaux sont «consacrés", ils protègent le bétail et attirent la bonne fortune. Ce chapitre se termine en incluant la chasse et la pêche, des activités très souvent liées au pastoralisme en Asie centrale et septentrionale. Si la première est valorisée, mais d'un apport alimentaire limité, la deuxième au contraire, sans prestige, a une réelle importance alimentaire. On remarquera les particularités de la chasse à l'ours en Sibérie et au loup en Mongolie, qui renvoient au statut symbolique particulier de ces animaux dans ces régions.

9 Le dernier chapitre aborde les relations entre nomades et sédentaires, relations fondées sur la complémentarité et la rivalité. Pour les nomades, il est indispensable d'entretenir des échanges réguliers avec les populations sédentaires, pour assurer tout autant leur approvisionnement en produits agricoles et manufacturés que l'écoulement de leur production animale. En fait, des " pratiques mixtes » se sont développées, « fondées sur la circulation régulière des hommes, des biens et des idées entre les territoires de 
parcours, les villages et les villes» (p.230). Au cours de la période contemporaine, l'abandon du mode de vie nomade a connu une intensification radicale en Asie centrale et septentrionale. La partie intitulée "le nomadisme entre essor et déclin " présente une chronologie et une géographie de l'alternance de ces périodes, en relation avec les sociétés sédentaires d'Eurasie. Après l'évocation de l'Âge du bronze en Asie centrale (E. Luneau), est abordée la domination des steppes eurasiatiques par les nomades, entre l'Âge du fer et l'expansion russe et chinoise (J. Thorez). La supériorité militaire des sociétés nomades, appuyée sur la maîtrise de la cavalerie, dura ainsi jusqu'au XVI ${ }^{\mathrm{e}}$ siècle et l'acquisition des techniques de l'artillerie par les États sédentaires, ce qui provoqua le déclin politique des populations nomades au profit des pouvoirs russe et chinois qui imposèrent alors leur domination. "Du nomadisme au capitalisme » rappelle qu'aux $\mathrm{XIX}^{\mathrm{e}}$ et $\mathrm{Xx}^{\mathrm{e}}$ siècles, les projets de modernisation liés à la colonisation russe puis à l'application du projet soviétique ont souvent été associés à des politiques de sédentarisation. Un exemple frappant analyse les temps coloniaux de la sédentarisation dans les steppes kazakhes, puis le processus de sédentarisation accéléré de la période de collectivisation de l'agriculture sous Stalin, ayant entraîné une famine meurtrière et l'exode des nomades du Kazakhstan (I. Ohayon). À la disparition de l'URSS s'ouvre une ère nouvelle de transformation économique, avec l'adoption d'une idéologie capitaliste; les conséquences de la décollectivisation et des privatisations sur l'économie pastorale sont examinées à partir de quelques exemples. «Le nomadisme dans un monde industriel et urbain » expose comment, durant la période soviétique, la création de régions industrielles et l'immigration de populations russophones ont souvent marginalisé les groupes nomades dans leur région d'origine, créant de réels déséquilibres démographiques (J.Thorez). L'extraction et la transformation d'hydrocarbures et de minerais ne cessent d'empiéter sur les territoires nomades et génèrent des pollutions nocives (en particulier le traitement du nickel à Noril'sk), de même que des feux de forêts, etc. Si l'urbanisation est un phénomène très fort dans de nombreuses régions, pour les Nénetses du Yamal, en Russie, il demeure une grande fluidité dans les relations entre la toundra et la ville, assortie d'une grande capacité d'adaptation (A. Besacier-Picard). En Mongolie, depuis la fin de la période socialiste, les nomades sédentarisés ont développé des "quartiers de yourtes " ou "de palissades " en périphérie des agglomérations, notamment à Oulan Bator, capitale très polluée qui concentre près de la moitié de la population du pays (G. Lacaze). Enfin, avec "L'héritage nomade en métamorphoses", l'ouvrage s'achève sur le constat que "si le nomadisme n'est plus le mode de vie dominant en Asie centrale et septentrionale, il demeure une référence majeure dans la rhétorique officielle et les pratiques quotidiennes » (p. 267). Les États lui assignent un rôle important dans la construction des identités nationales, comme au Kirghizstan, ou en Mongolie avec la figure de Gengis Khan érigé en héros nomade contemporain (N. Kradin). Par ailleurs, à la faveur de l'écotourisme qui s'est développé depuis environ deux décennies, les traditions nomades ont été restaurées dans plusieurs pays.

10 On ne peut que recommander la découverte de ce bel ouvrage foisonnant par la diversité de ses approches et, de ce fait, passionnant. 


\section{AUTEURS}

JOËLLE ROBERT-LAMBLIN

UPR 2147, CNRS 